\title{
Orbit equivalence and rigidity of ergodic actions of Lie groups
}

\author{
ROBERT J. ZIMMER $\dagger$ \\ From the Department of Mathematics, University of Chicago, USA
}

(Received 2 November 1980)

\begin{abstract}
The rigidity theorem for ergodic actions of semi-simple groups and their lattice subgroups provides results concerning orbit equivalence of the actions of these groups with finite invariant measure. The main point of this paper is to extend the rigidity theorem on one hand to actions of general Lie groups with finite invariant measure, and on the other to actions of lattices on homogeneous spaces of the ambient connected group possibly without invariant measure. For example, this enables us to deduce non-orbit equivalence results for the actions of SL $(n, \mathbb{Z})$ on projective space, Euclidean space, and general flag and Grassman varieties.
\end{abstract}

\section{Introduction}

If $G$ and $G^{\prime}$ are locally compact separable groups acting ergodically on measure spaces $(S, \mu),\left(S^{\prime}, \mu^{\prime}\right)$ respectively, the actions are called orbit equivalent if there exists (possibly after discarding null sets) a measure class preserving Borel bijection that takes $G$-orbits onto $G^{\prime}$-orbits. For actions of amenable groups, orbit equivalence is a very weak notion as one now has the result that any two free ergodic actions of amenable groups with finite invariant measure are orbit equivalent as long as both groups are either discrete or continuous and unimodular [16], [3]. On the other hand, we recently showed in [24] that for free irreducible ergodic actions of semi-simple Lie groups with finite centre and real rank at least 2, orbit equivalence completely determines the group up to local isomorphism and, in the centrefree case, completely determines the action as well up to an automorphism of the group. As explained in [24], this result for semi-simple groups is a direct analogue in ergodic theory of the rigidity theorems for lattices in semi-simple Lie groups of G. D. Mostow [15] and G. A. Margulis [10]-[12]. In fact, a basic ingredient of the proof of the rigidity theorem for ergodic actions in [24] is the use of a technique of Margulis for showing the rationality of certain measurable maps between algebraic varieties.

The results of [24] can be applied to actions of lattice subgroups and, for example, we deduce in $[24]$ that the actions of $\operatorname{SL}(n, \mathbb{Z})$ on the torus $\mathbb{R}^{n} / \mathbb{Z}^{n}$ are mutually non-orbit equivalent as we vary $n$, for $n \geq 2$. On the other hand, the results of [24]

$\dagger$ Address for correspondence: Dr Robert J. Zimmer, Department of Mathematics, University of Chicago, Chicago, Ill. 60637, USA. 
are strongly dependent upon the actions in question having finite invariant measure and thus do not apply to many natural and classical examples, for instance the action of $\operatorname{SL}(n, \mathbb{Z})$ on projective space and more general Grassman and flag varieties. The main point of this paper is to extend the rigidity theorem of [24] to enable us to deduce non-orbit equivalence results for this situation, and more generally for the ergodic actions of lattices in semi-simple Lie groups defined by translation on homogeneous spaces of these Lie groups. As an example of our results, we have the following. (See $\$ 4$ for a more general formulation.)

THEOREM 4.2. (a) The actions of $\operatorname{SL}(n, \mathbb{Z})$ on the projective spaces $\mathbb{P}^{n-1}$ are mutually non-orbit equivalent as $n$ varies, $n \geq 2$.

(b) The actions of $\operatorname{SL}(n, \mathbb{Z})$ on $\mathbb{R}^{n}$ are mutually non-orbit equivalent as $n$ varies, $n \geq 2$.

(c) For a fixed $n \geq 4$, let $G_{n, k}$ be the Grassman variety of $k$-planes in $\mathbb{R}^{n}$. Then the actions of $\mathrm{SL}(n, \mathbb{Z})$ on $G_{n, k}$ are mutually non-orbit equivalent as $k$ varies, $1 \leq k \leq[n / 2]$.

The extension of the rigidity theorem upon which these results depend is a result concerning orbit equivalence of ergodic actions of general (i.e. not necessarily semi-simple) Lie groups with finite invariant measure.

We recall that in any locally compact separable group $H$ there is a unique maximal normal closed amenable subgroup $N$. In connected groups, for example, this subgroup can be explicitly described as follows. Let $K$ be a compact normal subgroup such that $H / K$ is a Lie group. Let $R$ be the radical of $H / K$ and $Z$ the centre of $(H / K) / R$. Then

$$
((H / K) / R) / Z \cong \prod_{1}^{n} G_{i}
$$

where $G_{i}$ are connected centreless simple Lie groups. Letting

$$
\prod_{1}^{n} G_{i} \rightarrow \prod_{1}^{k} G_{i}
$$

be projection onto the product of the non-compact factors, we obtain a homomorphism $H \rightarrow \prod_{1}^{k} G_{i}$. The kernel of this homomorphism is the group $N$. Our first extension of the rigidity theorem to connected groups is the following.

TheOREM 3.1. Suppose $H, H^{\prime}$ are connected locally compact second countable groups, $N, N^{\prime}$ the maximal normal closed amenable subgroups, and that the real rank of every simple component of $H / N$ is at least 2. Suppose $S, S^{\prime}$ are (essentially) free ergodic $H, H^{\prime}$-spaces, respectively, with finite invariant measure and that the actions are orbit equivalent. Then $H / N$ and $H^{\prime} / N^{\prime}$ are isomorphic and $N$ is compact if and only if $N^{\prime}$ is compact.

One can actually weaken the assumption that the real rank of every simple component of $H / N$ is at least 2 to the assumption that the real rank of $H / N$ itself be at least 2 if one makes in addition certain irreducibility assumptions on the ergodic action of $H$ on $S$, which, for example, will always hold if the restriction of 
the $H$-action to $N$ is still ergodic. (See theorem 3.3 below.) As in [24], the assumption on the $\mathbb{R}$-rank is necessary in order to employ Margulis' techniques for proving the rationality of appropriate measurable maps. For results in a similar direction in the $\mathbb{R}$-rank one case, the reader is referred to [26] where Mostow's results on quasi-conformal mappings [14] are applied. When both the groups and actions are direct products, we obtain the following sharper result.

Theorem 3.4. Let $A, A^{\prime}$ be amenable groups, $G_{i}, G_{j}^{\prime}, i=1, \ldots, n, j=1, \ldots, p, b e$ connected semi-simple centreless Lie groups of $\mathbb{R}$-rank at least 2 , and suppose that neither $G_{i}$ and $G_{i}$ nor $G_{i}^{\prime}$ and $G_{i}^{\prime}$ have common factors for $i \neq j$. Let $S_{i}\left(S_{j}, T, T^{\prime}\right)$ be a free ergodic $G_{i}\left(G_{j}^{\prime}, A, A^{\prime}\right)$-space with finite invariant measure and suppose $S_{i}, S_{i}^{\prime}$ are irreducible. Let $X=T \times \prod S_{i}\left(Y=T^{\prime} \times \prod S_{i}^{\prime}\right)$ be the product ergodic $A \times \prod G_{i}\left(A^{\prime} \times \prod G_{j}^{\prime}\right)$-space. Suppose the actions are orbit equivalent and that either

(a) $A$ and $A^{\prime}$ are compact, or

(b) the $\mathbb{R}$-rank of every simple ergodic component of each $G_{i}$ is at least 2 .

Then

(i) $n=p$ and by reordering the indices we have $G_{i} \cong G_{i}^{\prime}$.

(ii) The $G_{i}$ actions on $S_{i}$ and $S_{i}^{\prime}$ are conjugate modulo an automorphism of $G_{i}$.

(iii) Under assumption (b), $\boldsymbol{A}$ is compact if and only if $\boldsymbol{A}^{\prime}$ is compact.

As in [24], these theorems can be applied to yield non-orbit equivalence theorems for ergodic actions of lattices in connected groups. See, for example, $\$ 4$ below.

The proofs of these theorems are based on a cohomological result which is a generalization of the cohomological result that is at the basis of the strong rigidity theorem proved in [24]. The proof of the present cohomological result in turn closely follows the proof in [24], with additional arguments needed to deal with the present more general hypotheses. In order to deduce theorem 3.4 , we also make use of a vanishing theorem for cohomology of ergodic actions of semi-simple Lie groups proved in [25] by different techniques (those of representation theory).

$\S 2$ below contains the proof of the cohomological result and $\S 3$, the proofs of the theorems for connected groups stated above. $\S 4$ contains the applications to (not necessarily measure preserving) actions of lattices.

\section{The main cohomological result}

$\S 2$ of [24] provides a brief summary of some of the notions from ergodic theory that we shall need. Here we shall only recall the notion of a cocycle and its connection to orbit equivalence, referring the reader to [24] and the references there for further background.

Suppose $(S, \mu)$ is an ergodic $G$-space where $\mu(S)=1$ and $G$ is locally compact and separable. If $M$ is a standard Borel group, a Borel function $\alpha: S \times G \rightarrow M$ is called a cocycle if, for all $g, h \in G$,

$$
\alpha(s, g h)=\alpha(s, g) \alpha(s g, h) \quad \text { a.e. }
$$

Two cocycles $\alpha, \beta: S \times G \rightarrow M$ are called equivalent if there is a Borel function $\phi: S \rightarrow M$ such that, for each $g$,

$$
\phi(s) \alpha(s, g) \phi(s g)^{-1}=\beta(s, g) \text { a.e. }
$$


Cocycles arise naturally in a variety of questions, in particular in problems concerning orbit equivalence.

Suppose $S, S^{\prime}$ are free ergodic $G, G^{\prime}$ spaces respectively and that the actions are orbit equivalent. (By 'free' here, we mean essentially free, i.e. almost all stabilizers are trivial.) Then, possibly after discarding Borel null sets, there is a measure class preserving Borel isomorphism $\theta: S \rightarrow S^{\prime}$ which takes orbits onto orbits. Define $\alpha: S \times G \rightarrow G^{\prime}$ by

$$
\theta(s) \cdot \alpha(s, g)=\theta(s g) .
$$

This is well defined by the freeness of the action and it is straightforward to check that $\alpha$ is a cocycle. If $\pi: G \rightarrow G^{\prime}$ is a homomorphism, one also obtains a cocycle $\beta: S \times G \rightarrow G^{\prime}$ simply by defining

$$
\beta(s, g)=\pi(g) \text {. }
$$

Then $\beta$ is called the restriction of $\pi$ to $S \times G$. A basic connection between orbit equivalence and the cocycle $\alpha$ is the following result proved in [24, proposition 2.4]. (For more details of the technical aspects of the proof in [24] see lemma 3.5 below.) We recall that ergodic $G$-spaces $S, S^{\prime}$ are called conjugate if, possibly after discarding invariant Borel null sets, there is a measure-class preserving Borel bijection $S \rightarrow S^{\prime}$ which is a $G$-map, and are called automorphically conjugate if the action on $S$ is conjugate to the $G$-action on $S^{\prime}$ defined by $(s, g) \rightarrow s \cdot A(g)$, where $A$ is an automorphism of $G$.

Proposition 2.1 [24, proposition 2.4]. Suppose $S, S^{\prime}$ are orbit equivalent $G$-spaces and $\alpha: S \times G \rightarrow G$ is the cocycle corresponding to an orbit equivalence. If $\alpha$ is equivalent to the restriction of an automorphism of $G$, then the actions are automorphically conjugate. If the automorphism is inner, the actions are conjugate.

If $\alpha: S \times G \rightarrow H$, where $H$ is a locally compact group, there is a naturally associated ergodic $H$-space $X$ called the Mackey range of $\alpha$ [9], [24]. If $\alpha$ is a cocycle coming from an orbit equivalence, the Mackey range is just the orbit equivalent $H$-space. If $H$ is a real algebraic group, any ergodic action has an 'algebraic hull' which is a conjugacy class of algebraic subgroups [23], [24]. This conjugacy class of algebraic subgroups is characterized by the property that the ergodic action in question is induced from an action of an algebraic subgroup if and only if the subgroup is contained in a member of the conjugacy class. An action is called Zariski-dense if the algebraic hull is $H$ itself. If $H$ is a Zariski-connected real algebraic gfoup with no compact factors, then the Borel density theorem [1] implies that any ergodic action of $H$ with finite invariant measure is Zariski-dense [23, theorem 1.4]. The main cohomological result we shall need is the following.

THEOREM 2.2. Let $H$ be a locally compact group, $N$ the maximal normal amenable subgroup of $H, G=H / N$, and suppose that $G$ is a connected semi-simple Lie group. (For example, take $H$ connected.) Suppose further than each of the simple components of $G$ has $\mathbb{R}$-rank at least 2 . Let $G^{\prime}$ be a connected semi-simple centrefree Lie group with no compact factors and suppose $G^{\prime} \subset \tilde{G}^{\prime}$, where $\tilde{G}^{\prime}$ is a Zariski-connected real 
algebraic group with trivial centre and $G^{\prime}$ of finite index in $\tilde{G}^{\prime}$. Let $\alpha: S \times H \rightarrow G^{\prime}$ be a cocycle with Zariski-dense range. Then there is a surjective homomorphism $\beta: G \rightarrow G^{\prime}$ such that $\alpha$ is equivalent to the restriction of $\beta \circ p$ to $S \times H$, where $p: H \rightarrow G$ is the natural projection.

This theorem is, of course, a generalization of [24, theorem 4.1]. There are two respects in which the hypotheses in [24] are weakened. First, we are considering cocycles defined on an $H$-space, where $H$ is considerably more general than a semi-simple group. Secondly, we are making no irreducibility assumptions on the ergodic action. Thus, for example, in the case in which $H=G=\prod G_{i}$, we do not assume each $G_{i}$ acts ergodically. We do assume, however, that the $\mathbb{R}$-rank of each $G_{i}$ is at least 2 . In [24], with an irreducibility assumption, we needed to assume only that the $\mathbb{R}$-rank of $G$ was at least 2 . One can formulate the theorem so as to subsume both situations but the statement is somewhat cumbersome and so we postpone presenting it until after the proof of theorem 2.2. However, the modifications of the arguments of [24, theorem 4.1] which are needed to prove the result in the more general form are all present in the proof of theorem 2.2.

Proof. We shall, in general, follow the proof of [24, theorem 4.1], referring the reader there for those parts of the argument that carry through without essential modification. Let $G=\prod_{1}^{k} G_{i}$, where $G_{i}$ are connected simple centreless Lie groups of $\mathbb{R}$-rank at least 2 . Let $P_{i}$ be a minimal parabolic subgroup of $G_{i}, P=\prod P_{i}$, and $\tilde{P}^{\prime}$ a minimal parabolic subgroup of $\tilde{G}^{\prime}$. Let $B=p^{-1}(P)$. Then $B$ is a minimal boundary subgroup of $H$ in the sense of Furstenberg [7] and is a maximal amenable (non-normal except in degenerate cases) subgroup of $H$. We have $N \subset B \subset H$ and $H / B=G / P$ as $H$-spaces. The action of $N$ on $G / P$ is, of course, trivial.

LEMMA 2.3. $S \times G / P$ is an ergodic $H$-space.

Proof. $S \times G / P=S \times H / B$ and by [21, theorem 4.2] $H$ is ergodic on this product if and only if $B$ is ergodic on $S$. Let $(E, \nu)$ be the space of ergodic components of the action of $N$ on $S$. Then there is an induced action of $G$ on $(E, \nu)$ and this is clearly measure preserving and ergodic, the latter since $H$ is ergodic on $S$. To see that $B$ is ergodic on $S$ it clearly suffices to show that $B / N=P$ is ergodic on $E$. However, this follows from Moore's ergodicity theorem [13].

We now remark that the $H$-space $S \times G / P=S \times H / B$ is an amenable ergodic $H$-space in the sense of [20]. This follows from the amenability of $B$ as in [24]. Arguing exactly as in [24] we see that there is an amenable algebraic subgroup $J^{\prime} \subset \tilde{G}^{\prime}$ (this is denoted by $H^{\prime}$ in [24]) and a measurable map $\phi: S \times G / P \rightarrow \tilde{G}^{\prime} / J^{\prime}$ such that for each $h \in H$ and almost all $(s, x) \in S \times G / P$,

$$
\phi(s h, x h) \cdot \alpha(s, h)^{-1}=\alpha(s, x) \text {. }
$$

We now show that $[\mathbf{2 4}$, lemma 4.2$]$ is still valid.

LEMMA 2.4. For almost all $s, \phi_{s}: G / P \rightarrow \tilde{G}^{\prime} / J^{\prime}$ defined by $\phi_{s}(x)=\phi(s, x)$ is a rational mapping. 
Proof. Let $A_{i}$ be a maximal $\mathbb{R}$-split connected Abelian subgroup of $P_{i}$. Fix $i$ and suppose $t \in A_{i}, t \neq e$. Let $H_{i}=p^{-1}\left(G_{i}\right)$ and $Y_{i}$ be the space of ergodic components of the $H_{i}$ action on $S$. Thus, for almost all $y \in Y_{i}$, we have $H_{i}$ is ergodic on $\left(S_{y}, \mu_{y}\right)$, where the latter is an ergodic component. Then for almost all $y \in Y_{i}$ we have, for all $h \in H_{i}$, that

$$
\phi(s h, x h) \cdot \alpha(s, h)^{-1}=\phi(s, x)
$$

for almost all $(s, x) \in S_{y} \times G / P$. We can then view $\phi$ as a map from $S_{y} \times G \rightarrow \tilde{G}^{\prime} / J^{\prime}$. Let $\hat{G}_{i}=\prod_{i \neq i} G_{j}$ and $\hat{P}_{i}=\prod_{i \neq i} P_{i}$; we shall then write $G=G_{i} \times \hat{G}_{i}$ and similarly for $P$. Since $H_{i}$ acts trivially on $\hat{G}_{i}$, we have, for all $h \in H_{i}$, that for almost all $(s, g, \hat{g}) \in S_{y} \times G_{i} \times \hat{G}_{i}$,

$$
\phi(s h, g h, \hat{g}) \cdot \alpha(s, h)^{-1}=\phi(s, g, \hat{g}) .
$$

Let $C$ be the centralizer of $t \in G_{i}$. Define for $(s, g) \in S_{y} \times G_{i}$ and $\hat{g} \in \hat{G}_{i}, w_{(s, g)}: C \rightarrow \tilde{G}^{\prime} / J^{\prime}$ by

$$
w_{(s, \hat{R}), \hat{\mathrm{k}}}(c)=\phi(s, c g, \hat{g}) .
$$

Define $T, \Sigma, \hat{\Sigma}, \tilde{w}$ as in [24]. Arguing exactly as in [24], we can view $w$ as a map from $S_{y} \times G_{i} / T \times \hat{G}_{i} \rightarrow \Sigma$ and again as in [24] we have that the map $\tilde{w}$ is $H_{i}$-invariant. We now claim that $H_{i}$ is ergodic on $S_{y} \times G_{i} / T$. The latter can also be expressed as $S_{y} \times H_{i} / p^{-1}(T)$ and so, by [21, theorem 4.2], it suffices to see that $p^{-1}(T)$ is ergodic on $S_{y}$. Let $E$ be the space of ergodic components of $N$ acting on $S_{y}$. As in lemma 2.3 there is an induced ergodic action of $G_{i}=H_{i} / N$ on $E$ preserving a finite measure. To see that $p^{-1}(T)$ is ergodic on $S_{y}$, it suffices to see that $T$ is ergodic on $E$, which follows from Moore's theorem [13]. With the ergodicity of $H_{i}$ on $S_{y} \times G_{i} / T$ established, one can argue exactly as in [24], fixing $y \in Y_{i}$ and $\hat{g} \in G_{i}$. Namely, suppose that $G_{i} \subset \tilde{G}_{i}$ is a subgroup of finite index, where $\tilde{G}_{i}$ is a Zariski-connected real algebraic group with trivial centre, and that $P_{i}=G_{i} \cap \tilde{P}_{i}$, where $\tilde{P}_{i}$ is a minimal parabolic in $\tilde{G}_{i}$. Let $U_{i}$ be the intersection with $G_{i}$ of the unipotent radical of a parabolic in $\tilde{G}_{i}$ opposite to $\tilde{P}_{i}$. Then the arguments of [24, lemma 4.2] and [11, p. 43] show that for almost all $y \in Y_{i}, s \in S_{y}, g \in G_{i}$, and $\hat{g} \in \hat{G}_{i}, \phi(s, u g, \hat{g})$ depends rationally on $u \in U_{i}$. By the definition of $Y_{i}$, this implies that $\phi(s, u g, \hat{g})$ depends rationally on $u \in U_{i}$ for almost all $(s, g, \hat{g}) \in S \times G_{i} \times \hat{G}_{i}$. Letting $i$ vary, we have that for almost all $s \in S, g_{1}, \ldots, g_{k} \in G$,

$$
\phi\left(s, u_{1} g_{1}, \ldots, u_{k} g_{k}\right)=\phi\left(s,\left(u_{1}, \ldots, u_{k}\right) \cdot\left(g_{1}, \ldots, g_{k}\right)\right)
$$

depends rationally on each $u_{i}$ for almost all $u_{j}$, for any $j \neq i$. Applying Margulis' result that a measurable function on $\mathbb{R}^{n} \times \mathbb{R}^{p}$ which is rational in $x$ for almost all $y$ and rational in $y$ for almost all $x$ must be rational [11, p. 43], [12], we deduce that for almost all $s \in S, g \in G, \phi(s, u g)$ is rational in $u$ for $u \in \Pi U_{i}$. Arguing as in [11, p. 43], an application of $[2,4.10]$ completes the proof of the lemma.

The remainder of the proof of [24, theorem 4.1] now carries over with only minor modifications, and we make only one further observation. Define $\Phi_{0}$ and $\beta$ as in [24]. Then one obtains a homomorphism $\beta: H \rightarrow \tilde{G}^{\prime}$ such that

$$
\Phi_{0} \cdot \beta(h)=\Phi_{0} \cdot p(h) \text {. }
$$


In particular, for $h \in N, \beta(h)$ leaves $\Phi_{0}(G / P)$ pointwise fixed. Arguing as in [24] (where it is shown that $\beta$ as first defined is independent of $s$ ), we obtain that $\beta$ factors to a homomorphism $G \rightarrow \tilde{G}^{\prime}$. This observation and the arguments of [24] then complete the proof of theorem 2.2.

We recall that a semi-simple Lie group acts irreducibly on a probability space if the restriction to each non-central normal subgroup is ergodic. Basically the same proof as above, using irreducibility as in [24, theorem 4.1], shows the following variant of theorem 2.2. In certain situations it allows one to pass from the assumption that every simple component of $H / N$ has $\mathbb{R}$-rank at least 2 to the assumption that $H / N$ has $\mathbb{R}$-rank at least 2 .

THEOREM 2.5. Let $H$ be a locally compact separable group, $N$ the maximal normal amenable subgroup and suppose $H / N=G$ is a product $\Pi G_{i}$ of semi-simple Lie groups with $\mathbb{R}$-rank of $G_{i}$ at least 2 . Suppose further that each $G_{i}$ acts irreducibly on almost all ergodic components of its action on $E$, where the latter is the space of ergodic components of the N-action. Let $G^{\prime}, \alpha$ be as in theorem 2.2. Then the conclusion of theorem 2.2 is true.

The hypotheses of theorem 2.5 hold in particular if $N$ is already ergodic on $S$. They are also satisfied if $H=N \times G, G$ acts irreducibly on $S$, and the $\mathbb{R}$-rank of $G$ is at least 2. The latter includes the situation dealt with in [24].

\section{Rigidity theorems}

In this section, we prove the theorems for connected groups stated in the introduction.

THeOREM 3.1. Suppose $H, H^{\prime}$ are locally compact separable groups, $N, N^{\prime}$ the maximal normal amenable subgroups, and suppose $G=H / N$ and $G^{\prime}=H^{\prime} / N^{\prime}$ are connected (or equivalently, that $G$ and $G^{\prime}$ are centrefree semi-simple connected Lie groups). Suppose further that the $\mathbb{R}$-rank of every simple component of $G$ is at least 2. Let $S, S^{\prime}$ be free ergodic $H, H^{\prime}$-spaces, respectively, with finite invariant measure, and suppose that the actions are orbit equivalent. Then $G$ and $G^{\prime}$ are isomorphic and $N$ is compact if and only if $N^{\prime}$ is compact.

We prepare the proof by recalling some results we shall need. If $E$ is a subset of a space $X$ on which a group acts, its saturation $[E]$ is the union of all orbits intersecting $E$. If $E \subset A \subset X$, we shall call $E$ saturated in $A$ if $[E] \cap A=E$. In particular, $E$ is saturated in $X$ if and only if it is invariant. A Borel subset of $X$ is called a countable section if it intersects every orbit in at most countably many points, and the countable section is called complete if its saturation is conull [6]. Every action of a locally compact separable group on a standard measure space has a complete countable section [6, theorem 2.8]. Furthermore, there exists a measure class on the countable section whose null sets are precisely the negligible sets (contained in the section) [17, theorem 6.17], [6, prop. 3.6, 3.7]. (We recall that a set is negligible if its saturation is null.) This measure class on the section has the further property that it is invariant under the partial automorphisms of the 
countable equivalence relation defined on it [5]. It is also not difficult to see that one can choose the section to lie inside any predetermined conull set. We also recall that if a group $G$ acts on a standard measure space $(S, \mu)$, by an inessential reduction of the action we mean a subset

$$
S_{0} * G=\left\{(s, g) \in S \times G \mid s, s g \in S_{0}\right\},
$$

where $S_{0}$ is conull. A basic technical fact proven by A. Ramsay [17, lemma 5.2] is the following.

LEMMA 3.2. If $U \subset S \times G$ is conull, Borel, and satisfies $(s, g),(s g, h) \in U$ implies $(s, g h) \in U$, then $U$ contains an inessential reduction.

We now turn to the proof of theorem 3.1.

Proof of theorem 3.1. Let $\theta: S \rightarrow S^{\prime}$ be an orbit equivalence and $\alpha: S \times H \rightarrow H^{\prime}$ the corresponding cocycle. Let $p^{\prime}: H^{\prime} \rightarrow G^{\prime}$ be the natural projection. The Mackey range of $\alpha$ is just the $H^{\prime}$ action on $S^{\prime}$ and it is not difficult to verify that the Mackey range of $p^{\prime} \circ \alpha$ is then just the $G^{\prime}$ action induced on the space of ergodic components of the $N^{\prime}$ action on $S^{\prime}$. But this $G^{\prime}$ action has finite invariant measure and hence the Mackey range of $p^{\prime} \circ \alpha$ is Zariski-dense in $G^{\prime}$ [23, theorem 1.4]. It follows from theorem 2.2 that $p^{\prime} \circ \alpha$ is equivalent to the restriction of a surjective homomorphism $\beta: H \rightarrow G^{\prime}$ which is trivial on $N$. With the hypothesis of theorem 3.1, one can now quickly deduce that $G$ and $G^{\prime}$ are isomorphic by observing that the existence of a surjective homomorphism $G \rightarrow G^{\prime}$ implies that the $\mathbb{R}$-rank of every simple component of $G^{\prime}$ must be at least 2 and so one can apply theorem 2.2 to the cocycle coming from $\theta^{-1}$ as well. However, we shall present an alternative proof which will simultaneously yield the conclusion about $N$ and $N^{\prime}$ and generalize immediately to provide a proof of theorem 3.3 below.

Let $K$ be the kernel of $\beta$, so $N \subset K \subset H$. Let $\lambda: S \rightarrow G^{\prime}$ be a Borel function such that for each $h \in H$,

$$
\lambda(s) p^{\prime}(\alpha(s, h)) \lambda(s h)^{-1}=\beta(h)
$$

for almost all $s \in S$. Composing $\lambda$ with a Borel section of $p^{\prime}$ we obtain a Borel function $\tilde{\lambda}: S \rightarrow H^{\prime}$ such that, for all $h \in H$,

Define $f: S \rightarrow S^{\prime}$ by

$$
p^{\prime}\left(\tilde{\lambda}(s) \alpha(s, h) \tilde{\lambda}(s h)^{-1}\right)=\beta(h) \quad \text { a.e. }
$$

$$
f(s)=\theta(s) \cdot \tilde{\lambda}(s)^{-1} .
$$

By lemma 3.2 and the definition of orbit equivalence we can find an inessential reduction $S_{0} * H$ of the $H$-action on $S$ such that:

(i) For all $(s, h) \in S_{0} * H, \lambda(s) p^{\prime}(\alpha(s, h)) \lambda(s h)^{-1}=\beta(h)$.

(ii) $\alpha$ is strict on $S_{0} * H$, i.e. the cocycle identity holds for all $(s, g),(s g, h) \in S_{0} * H$.

(iii) $\theta(s h)=\theta(s) \cdot \alpha(s, h)$.

Now restrict the $H$-action to $K$ and choose a complete countable section $T$ for the $K$-action with $T \subset S_{0}$. We now claim that for $s, t \in S_{0}, s$ and $t$ are in the same $K$-orbit if and only if $f(s), f(t)$ are in the same $N^{\prime}$-orbit. If $s=t \cdot h$ for $h \in K$, then

$$
f(s)=\theta(t h) \cdot \tilde{\lambda}(t h)^{-1}=\theta(t) \alpha(t, h) \alpha(t, h)^{-1} \tilde{\lambda}(t)^{-1} n=f(t) \cdot n,
$$


for some $n \in N^{\prime}$. Conversely, suppose $f(s)=f(t) \cdot n$ for some $n \in N^{\prime}$. Then

$$
\theta(s) \tilde{\lambda}(s)^{-1}=\theta(t) \tilde{\lambda}(t)^{-1} n
$$

and hence $\theta(s)$ and $\theta(t)$ are in the same $H^{\prime}$ orbit. Since $\theta$ is an orbit equivalence, this implies $s, t$ are in the same $H$ orbit, so we have $t=s \cdot h$ for some $h \in H$. Thus

$$
\theta(s) \tilde{\lambda}(s)^{-1}=\theta(s h) \tilde{\lambda}(s h)^{-1} n
$$

and, by the definition of $\alpha$, we have

$$
\alpha(s, h)=\tilde{\lambda}(s)^{-1} n^{-1} \tilde{\lambda}(s h) .
$$

Thus $\beta(h)=e$, so $h \in K$.

Let $T^{\prime}=f(T)$. Let $\nu$ be a probability measure on $T$ whose null sets are the $K$-negligible sets and let $\nu^{\prime}=f_{*}(\nu)$, a measure on $T^{\prime}$. By discarding a $\nu^{\prime}$-null set of $T^{\prime}$, we can choose a Borel section $\sigma: T^{\prime} \rightarrow T$ of $f$. Let $T_{0}=\sigma\left(T^{\prime}\right)$ and $\nu_{0}=\sigma_{*}\left(\nu^{\prime}\right)$. Then one easily checks that $T_{0}$ is also a complete countable section and that $\nu_{0}$-null sets are the $K$-negligible sets contained in $T_{0}$. In other words, replacing $(T, \nu)$ by $\left(T_{0}, \nu_{0}\right)$, we may assume $f:(T, \nu) \rightarrow\left(T^{\prime}, \nu^{\prime}\right)$ and its inverse are measure preserving Borel isomorphisms which preserve the countable equivalence relations defined by the $K$ and $N^{\prime}$ actions respectively. Now let $d n^{\prime}$ be a probability measure on $N$ in the same measure class as Haar measure, and define

$$
m=\int\left(\nu^{\prime} \cdot n^{\prime}\right) d n^{\prime}
$$

Then $m$ is a measure quasi-invariant under $N^{\prime}$ and the $\nu^{\prime}$-null sets coincide with the $m$-negligible subsets of $T^{\prime}$ under the $N^{\prime}$-action. Since the $K$-action on $(S, \mu)$ and the $N^{\prime}$-action on $\left(S^{\prime}, m\right)$ have isomorphic (in the sense of countable ergodic equivalence relations [5]) complete countable sections, it follows from the results of [6] (e.g. propositions 3.6, 3.7, corollary 5.8) that the $K$-action on $(S, \mu)$ and the $N^{\prime}$ action on $\left(S^{\prime}, m\right)$ are orbit equivalent. The notion of an amenable ergodic action [20] can be extended without difficulty to the non-ergodic case and, since $N^{\prime}$ is amenable, the action on $\left(S^{\prime}, m\right)$ will be amenable [20, theorem 2.1]. Since the $K$-action on $(S, \mu)$ is free and orbit equivalent to the $N^{\prime}$-action, the $K$-action on $(S, \mu)$ is amenable. But this action has finite invariant measure and hence $K$ is amenable. By the definition of $N$, this implies $K=N$, and hence $\beta$ is injective on $G$. This proves the first assertion of the theorem.

To prove the second assertion of the theorem, suppose $N^{\prime}$ is compact. Then the action of $N^{\prime}$ on $S^{\prime}$ is smooth, i.e. $S^{\prime} / N^{\prime}$ is a standard Borel space, and we have an induced action of $G^{\prime}$ on $S^{\prime} / N^{\prime}$ which is clearly ergodic. Furthermore, by the results of [6] (theorem 2.8 and those quoted in the previous paragraph), the $G^{\prime}$ action on $S^{\prime} / N^{\prime}$ and the $H^{\prime}$ action on $S^{\prime}$ are orbit equivalent. In other words, we may assume $N^{\prime}=\{e\}$. However, as shown above, the $N$ action on $S$ is similar in the sense of [6] to the $N^{\prime}$ action on $S^{\prime}$ (since they have isomorphic complete countable sections). This implies that the $N$ action on $S$ is also type I, i.e. almost every ergodic component of the $N$ action on $S$ is a transitive $N$-space. But since the action on $S$ has finite invariant measure, and since the action is free, this implies that Haar 
measure on $N$ is finite. Hence, $N$ is also compact. This completes the proof of the theorem.

Using theorem 2.5 in place of theorem 2.2, one obtains the following generalization of theorem 3.1. The proof is basically the same.

THEOREM 3.3. Let $H, H^{\prime}$ be locally compact separable groups, $N, N^{\prime}$ the maximal normal amenable subgroups, $G=H / N, G^{\prime}=H^{\prime} / N^{\prime}$, and suppose $G, G^{\prime}$ connected. Let $S, S^{\prime}$ be free ergodic $H, H^{\prime}$-spaces respectively, with finite invariant measure, and suppose the actions are orbit equivalent. Let $E$ be the space of ergodic components of the $N$-action on $S$. Suppose further that $G=\prod G_{i}$ where each $G_{i}$ is a semi-simple Lie group of $\mathbb{R}$-rank at least 2 ; and that $G_{i}$ acts irreducibly on almost all of its ergodic components on $E$. Then $G$ and $G^{\prime}$ are isomorphic and $N$ is compact if and only if $N^{\prime}$ is compact.

When the groups are product groups and the actions are product actions, we deduce a sharper result.

TheOrem 3.4. Let $A, A^{\prime}$ be amenable groups, $G_{i}, G_{j}^{\prime}, i=1, \ldots, n, j=1, \ldots, p$, be connected semi-simple centrefree Lie groups of $\mathbb{R}$-rank at least 2 and suppose that neither $G_{i}$ and $G_{j}$ nor $G_{i}^{\prime}$ and $G_{j}^{\prime}$ have common factors for $i \neq j$. Let $S_{i}\left(S_{i}, T, T^{\prime}\right)$ be a free ergodic $G_{i}\left(G_{i}^{\prime}, A, A^{\prime}\right)$-space with finite invariant measure and suppose $S_{i}, S_{i}^{\prime}$ are irreducible. Let $X=T \times \prod S_{i}\left(Y=T^{\prime} \times \prod S_{i}^{\prime}\right)$ be the product ergodic $A \times \prod G_{i}\left(A^{\prime} \times \prod G_{i}^{\prime}\right)$-space. Suppose the actions are orbit equivalent and that either

(a) $A$ and $A^{\prime}$ are compact, or

(b) the $\mathbb{R}$-rank of every simple ergodic component of each $G_{i}$ is at least 2 . Then

(i) $n=p$ and by reordering the indices we have $G_{i}=G_{i}^{\prime}$.

(ii) The $G_{i}$ actions on $S_{i}$ and $S_{i}^{\prime}$ are conjugate modulo and automorphism of $G_{i}$.

(iii) Under assumption (b), $A$ is compact if and only if $A^{\prime}$ is compact.

Proof. We first remark that, since any amenable ergodic action is hyperfinite [16], [3], we can assume that $A$ is either the real line, the integers, or the identity (the latter in case $A$ is compact, using [6] as above). Let $G=\prod G_{i}, G^{\prime}=\prod G_{i}^{\prime}, \theta: X \rightarrow Y$ an orbit equivalence and $\alpha: X \times A \times G \rightarrow A^{\prime} \times G^{\prime}$ the corresponding cocycle. Write $\alpha=\left(\alpha_{1}, \alpha_{2}\right)$, where $\alpha_{i}$ is the composition of $\alpha$ with the projection on $A^{\prime}$ and $G^{\prime}$ respectively. By theorems 2.5 and 3.3, there is a function $\lambda: X \rightarrow G^{\prime}$ such that, for each $(a, g) \in A \times G$ and almost all $x \in X$,

$$
\lambda(x) \alpha_{2}(x, a, g) \lambda(x \cdot(a, g))^{-1}=\beta(g),
$$

where $\beta: G \rightarrow G^{\prime}$ is an isomorphism. If $A$ and $A^{\prime}$ are not the identity, then by hypothesis $(b)$ and [25, theorem $A]$, the cocycle $\alpha_{1} \mid X \times G$ is trivial. In other words, we can find $\phi: X \rightarrow A^{\prime}$ such that for all $g \in G$,

$$
\phi(x) \alpha_{1}(x, e, g) \phi(x \cdot(e, g))^{-1}=e \quad \text { for almost all } x .
$$

From this it follows that

$$
\phi(x \cdot(a, g))^{-1}=\phi((x \cdot(a, e)) \cdot(e, g))^{-1}=\alpha_{1}(x \cdot(a, e), e, g)^{-1} \phi(x \cdot(a, e))^{-1} .
$$


Define $f: X \rightarrow Y$ by

$$
f(x)=\theta(x) \cdot(\phi(x), \lambda(x))^{-1} .
$$

Then, for almost all $x \in X$ and $(a, g) \in H$, we have

$$
\begin{aligned}
f(x \cdot(a, g))=\theta(x \cdot(a, g))\left(\phi(x \cdot(a, g))^{-1}, \lambda(x \cdot(a, g))^{-1}\right) \\
\quad=\theta(x) \cdot \alpha(x, a, g)\left(\alpha_{1}(x \cdot(a, e), e, g)^{-1} \phi(x \cdot(a, e))^{-1}, \alpha_{2}(x, a, g)^{-1} \lambda(x)^{-1} \beta(g)\right) \\
\quad=\theta(x) \cdot\left(\alpha_{1}(x, a, e) \phi(x \cdot(a, e))^{-1}, \lambda(x)^{-1} \beta(g)\right) .
\end{aligned}
$$

Thus,

$$
f(x \cdot(a, g))=f(x) \cdot(\gamma(x, a), \beta(g)),
$$

where $\gamma(x, a)$ is the cocycle

$$
\phi(x) \alpha_{1}(x, a, e) \phi(x \cdot(a, e))^{-1} .
$$

Let $f=\left(f_{1}, f_{2}\right)$, where $f_{1}$ and $f_{2}$ are the compositions of $f$ with the projection of $Y$ onto $T^{\prime}$ and $S^{\prime}=\prod S_{i}^{\prime}$ respectively. Writing $x \in X=T \times S$ as $x=(t, s)$ we have

$$
f_{2}((t, s) \cdot(a, g))=f_{2}(t, s) \cdot \beta(g),
$$

and, setting $g=e$, we obtain

$$
f_{2}(t a, s)=f_{2}(t, s)
$$

For each $a \in A$, this equality holds for almost all $(t, s)$. Thus, for all $a$ in a countable dense subgroup of $A$, and for almost all $s$, this equality holds for almost all $t$. But a Borel function which is essentially invariant under a countable dense subgroup is actually essentially invariant under the entire group. (To see this one can, for example, argue as follows. Choose a Borel isomorphism of $S^{\prime}$ with $[0,1]$ so that the function is then identified with an element of $L^{\infty} \subset L^{2}$. Then use the continuity of the naturally induced representation on $L^{2}$.) Thus for $s$ in a conull set, for all $a \in A$, we have

$$
f_{2}(t a, s)=f_{2}(t, s) \quad \text { for almost all } t .
$$

By ergodicity of the $A$-action on $T$ this implies that $f_{2}$ is essentially independent of $t$, and so we can write

$$
f_{2}(t, s)=F_{2}(s) \quad \text { a.e., }
$$

where $F_{2}: S \rightarrow S^{\prime}$. Furthermore, we have

$$
f_{1}((t, s)(e, g))=f_{1}(t, s)
$$

and so a similar argument shows that

$$
f_{1}(t, s)=F_{1}(t) \text { a.e., }
$$

where $F_{1}: T \rightarrow T^{\prime}$. We thus have

$$
f(t, s)=\left(F_{1}(t), F_{2}(s)\right) \text { a.e. }
$$

We now show, via a technical argument, that $F_{2}$ is measure class preserving, essentially bijective, and defines a Boolean map $B\left(S^{\prime}\right) \rightarrow B(S)$ that behaves well with respect to the $G$ and $G^{\prime}$ actions.

We first remark that, for all $g$,

$$
F_{2}(s g)=F_{2}(s) \cdot \beta(g) \text { a.e. }
$$


By lemma 3.2 there is a conull Borel set $S_{1} \subset S$ such that this equality holds for all $(s, g) \in S_{1} * G$. By the definition of $f$ (and of orbit equivalence), there is a conull set in $T \times S$ such that, if $f(t, s)$ and $f\left(t^{\prime}, s^{\prime}\right)$ are in the same $A^{\prime} \times G^{\prime}$ orbit for $(t, s),\left(t^{\prime}, s^{\prime}\right)$ in this conull set, then $(t, s)$ and $\left(t^{\prime}, s^{\prime}\right)$ are in the same $A \times G$ orbit. Since $f=\left(F_{1}, F_{2}\right)$ a.e., the same statement is true if we replace $f$ by $\left(F_{1}, F_{2}\right)$. By Fubini's theorem, there is a conull Borel set $S_{2} \subset S$ such that, if $s, s^{\prime} \in S_{2}$ with $F_{2}(s)$ and $F_{2}\left(s^{\prime}\right)$ in the same $G^{\prime}$-orbit, then $s, s^{\prime}$ are in the same $G$ orbit. Let $S_{0}=S_{1} \cap S_{2}$. Then, if $s, s^{\prime} \in S_{0}$ with $F_{2}(s)=F_{2}\left(s^{\prime}\right)$, we have $s^{\prime}=s g$ for some $g \in G$ and so

$$
F_{2}\left(s^{\prime}\right)=F_{2}(s) \cdot \beta(g) \text {. }
$$

Since the $G^{\prime}$ action is free, $\beta(g)=e$, and since $\beta$ is an isomorphism it follows that $s=s^{\prime}$. Replacing $S_{0}$ by a conull Borel subset, we can assume that $S_{0}$ is invariant under a countable dense subgroup $G_{0} \subset G$.

Recall that the definition of orbit equivalence entails the existence of a conull Borel subset $(T \times S)_{0} \subset T \times S$ such that $\theta:(T \times S)_{0} \rightarrow \theta\left((T \times S)_{0}\right)$ is a measure class preserving Borel isomorphism onto its image which is conull in $T^{\prime} \times S^{\prime}$ and which takes orbits in $(T \times S)_{0}$ onto orbits in $\theta\left((T \times S)_{0}\right)$. In particular, viewing $\theta$ as a map $(T \times S)_{0} \rightarrow T^{\prime} \times S^{\prime}$, for an invariant set $D \subset T^{\prime} \times S^{\prime}$ we have $\theta^{-1}(D)$ is null in $T \times S$ if and only if $D$ is null in $T^{\prime} \times S^{\prime}$. By passing to a conull subset we can clearly also assume that $f=\left(F_{1}, F_{2}\right)$ on $(T \times S)_{0}$. If $B \subset T^{\prime} \times S^{\prime}$, then by the definition of $f$ for a point $y \in(T \times S)_{0}$, we have $f(y) \in[B]$ if and only if $\theta(y) \in[B]$, i.e. (taking $f$ to be also defined on $\left.(T \times S)_{0}\right)$ we have

$$
f^{-1}([B])=\theta^{-1}([B])
$$

Thus, if $B \subset T^{\prime} \times S^{\prime}$ is invariant, we have $B$ is null if and only if $\left(F_{1}, F_{2}\right)^{-1}(B) \cap$ $(T \times S)_{0}$ is null. Applying this to $B=T^{\prime} \times A$, where $A \subset S^{\prime}$ is invariant, we have that $A$ is null if and only if $\left(T \times F_{2}^{-1}(A)\right) \cap(T \times S)_{0}$ is null, which is clearly true if and only if $F_{2}^{-1}(A)$ is null. Since $A$ is invariant, $F_{2}^{-1}(A) \cap S_{0}$ is saturated in $S_{0}$ by the choice of $S_{0}$.

We shall now prove the following lemma, which we shall then apply to $F_{2}$. We also remark that this lemma spells out in more detail part of the argument of [24, proposition 2.4].

LEMMA 3.5. Suppose $\left(S_{i}, \mu_{i}\right), i=1,2$, are free ergodic $G$-spaces with finite invariant measure, where $G$ is a locally compact separable group. Suppose there is a conull Borel set $S_{0} \subset S_{1}$, and a Borel function $F: S_{0} \rightarrow S_{2}$ satisfying the following conditions:

(i) There is an automorphism $\beta$ of $G$ such that, for all $(s, g) \in S_{0} * G, F(s g)=$ $F(s) \cdot \beta(g)$.

(ii) $F$ is injective on $S_{0}$.

(iii) If $s, t \in S_{0}$ with $F(s)$ and $F(t)$ in the same orbit, then $s$ and $t$ are in the same orbit.

(iv) $S_{0}$ is invariant under a countable dense subgroup $G_{0} \subset G$.

(v) If $A \subset S_{2}$ is invariant, then $A$ is null if and only if $F^{-1}(A) \cap S_{0}$ is negligible. Then $F\left(S_{0}\right)$ is conull in $S_{2}$ and $F: S_{0} \rightarrow F\left(S_{0}\right)$ is a measure class preserving Borel isomorphism. 
Proof. The lemma will follow by an application of the local representation of an invariant measure as described by C. Series [18, prop. 1.1], [19, lemma 1.4]. Let $E$ be a complete countable section of the $G$-action on $S_{1}$, with $E \subset S_{0}$, such that $E$ is lacunary in the sense of [6]. (In the language of [19] $E$ is a sufficient regular transversal.) This means there is a neighbourhood $U$ of the identity in $G$ such that the product map $E \times U \rightarrow E \cdot U \subset S$ is injective. Let $\nu$ be a measure on $E$ whose null sets are the negligible (in $S_{1}$ ) subsets of $E$. Let $\lambda$ be Haar measure on $G$. Then by Series' results, identifying $E \times U$ with $E \cdot U$, the restriction of the measure $\mu$ to $E \cdot U$ is in the same measure class as the product measure $\nu \times \lambda$ on $E \times U$. For $y \in E$, let

$$
U_{y}=\left\{g \in U \mid y g \in S_{0}\right\} \text {, and } E_{0}=\left\{y \mid \lambda\left(U-U_{y}\right)=0\right\} .
$$

By Fubini's theorem, $E_{0}$ is a conull Borel subset of $E$. Let

$$
\left(E^{\prime}, \nu^{\prime}\right)=\left(F\left(E_{0}\right), F_{*}(\nu)\right) \text {. }
$$

By the hypothesis on $F, F\left(E_{0}\right)$ is a complete countable section for the $G$-action on $S_{2}$, and $F_{*}(\nu)$-null sets in $F\left(E_{0}\right)$ are the negligible subsets of $S_{2}$ contained in $F\left(E_{0}\right)$. We claim that the product map $F\left(E_{0}\right) \times \beta(U) \rightarrow S_{2}$ is injective. Suppose

$$
F\left(y_{1}\right) \cdot \beta\left(g_{1}\right)=F\left(y_{2}\right) \cdot \beta\left(g_{2}\right) \text {, }
$$

where $y_{i} \in E_{0}$ and $g_{i} \in U$. The set

$$
\beta\left(g_{1}\right) \beta\left(g_{2}\right)^{-1} \beta(U) \cap \beta(U)
$$

is clearly open and non-empty (as it contains $\beta\left(g_{1}\right)$ ). For $g$ in this set, let

$$
h(g)=\beta\left(g_{2}\right) \beta\left(g_{1}\right)^{-1} g \text {, }
$$

so that $h(g)$ is also in $\beta(U)$. We thus have for a set $g \in \beta(U)$ of positive measure,

$$
F\left(y_{1}\right) \cdot g=F\left(y_{2}\right) \cdot h(g) \text {. }
$$

Since $\beta$ is an automorphism of $G, \beta: U \rightarrow \beta(U)$ is measure class preserving. Thus we can find $a \in U_{y_{1}}$ such that $\beta^{-1} h \beta(a) \in U_{y_{2}}$ and such that $g=\beta(a)$ satisfies the above condition.

But since $y_{1}, y_{2}, y_{1} \cdot a, y_{2} \cdot \beta^{-1} h \beta(a) \in S_{0}$, we have

$$
F\left(y_{1} \cdot a\right)=F\left(y_{1}\right) \beta(a)=F\left(y_{2}\right) h(\beta(a))=F\left(y_{2} \cdot \beta^{-1} h \beta(a)\right) \text {. }
$$

As $F$ is injective on $S_{0}$,

$$
y_{1} \cdot a=y_{2} \cdot \beta^{-1} h \beta(a),
$$

which implies $y_{1}=y_{2}$ and $\beta(a)=h \beta(a)$. This implies that $\beta\left(g_{1}\right)=\beta\left(g_{2}\right)$, and injectivity of $F\left(E_{0}\right) \times \beta(U) \rightarrow S_{2}$ is thus established. Hence we can identify $F\left(E_{0}\right) \cdot \beta(U)$ with $F\left(E_{0}\right) \times \beta(U)$ and, by the results of Series mentioned above, the measure $\mu_{2}$ on the former agrees up to measure class with $F_{*}(\nu) \times \lambda$. This shows that $F:\left(E_{0} \cdot U\right) \cap S_{0} \rightarrow F\left(E_{0}\right) \cdot \beta(U)$ is a Borel bijection onto a conull subset of $F\left(E_{0}\right) \cdot \beta(U)$ and is measure class preserving. For any element $g \in G_{0}$ we have a commutative diagram (figure 1).

The conclusion of lemma 3.5 then follows by an elementary argument.

Returning to the proof of theorem 3.4, it follows by an application of lemma 3.5 that $F_{2}$ defines a Boolean $\sigma$-algebra isomorphism $B\left(S^{\prime}, \mu^{\prime}\right) \rightarrow B(S, \mu)$ which is a 


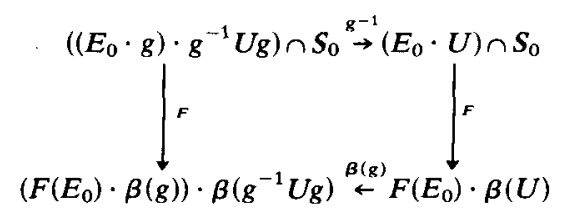

Figure 1

Boolean $G$-map when $G$ acts on $S^{\prime}$ via $s^{\prime} \cdot g=s^{\prime} \beta(g)$. Thus by [8] the actions of $G$ on $S$ and $S^{\prime}$ are conjugate. The isomorphism $\beta$ can be written as a product $\beta_{1} \times \cdots \times \beta_{n}$, where $\beta_{i}: G_{i} \rightarrow G_{1}^{\prime}$ is an isomorphism, possibly after reordering $G_{i}$. To complete the proof it clearly suffices to prove the following lemma.

Lemma 3.6. Suppose $G_{i}$ are locally compact groups, $S_{i}, S_{i}^{\prime}$ ergodic $G_{i}$-spaces, $G=$ $\prod G_{i}, S=\prod S_{i}, S^{\prime}=\prod S_{i}^{\prime}$. Suppose the $G$ actions on $S$ and $S^{\prime}$ are conjugate modulo an automorphism $\beta$ of $G$ of the form $\prod \beta_{i}$, where $\beta_{i}$ is an automorphism of $G_{i}$. Then the $G_{i}$ actions on $S_{i}$ and $S_{i}^{\prime}$ are conjugate modulo $\beta_{i}$.

Proof. Suppose $f: S \rightarrow S^{\prime}$ is such that

$$
f(s g)=f(s) \beta(g) \text { a.e. }
$$

Let $f_{i}$ be the coordinate functions of $f$ and suppose $g_{j} \in G_{j}, j \neq i$. Then

$$
f_{i}\left(s_{1} g_{1}, \ldots, s_{i}, \ldots, s_{n} g_{n}\right)=f_{i}\left(s_{1}, \ldots, s_{n}\right) \text {. }
$$

By ergodicity, $f_{i}$ is essentially independent of $s_{i}, j \neq i$, and so $f_{i}$ factors to a measure preserving map $S_{i} \rightarrow S_{i}^{\prime}$ such that

$$
f_{i}\left(s_{i} g_{i}\right)=f\left(s_{i}\right) \beta\left(g_{i}\right) \text { a.e. }
$$

Since $f=\prod f_{i}$ and $f$ is essentially injective so is each $f_{i}$, and this completes the proof.

\section{Applications to actions of lattices}

Suppose $H_{1}, H_{2}$ are closed subgroups of a locally compact group $G$. Then the orbit space of the action of $H_{1}$ on $G / H_{2}$ and that of $H_{2}$ on $G / H_{1}$ can both be naturally identified with the space of double cosets. It is, of course, possible that $\mathrm{G} / \mathrm{H}_{2}$ has a finite invariant measure while $G / H_{1}$ does not. We may thus hope to use results about orbit equivalence of actions with finite invariant measure to obtain results about certain actions which have no invariant measure. (Similar ideas have been used many times before. See, for example, [13], [21].)

THEOREM 4.1. Let $G, G^{\prime}$ be connected semi-simple Lie groups with finite centre, and $H \subset G, H^{\prime} \subset G^{\prime}$ almost connected non-compact subgroups. Let $\Gamma \subset G, \Gamma^{\prime} \subset G^{\prime}$ be irreducible lattices and suppose the $\Gamma$-action on $G / H$ and the $\Gamma^{\prime}$-action on $G^{\prime} / H^{\prime}$ are essentially free and orbit equivalent. Let $N, N^{\prime}$ be the maximal normal amenable subgroups of $H, H^{\prime}$ respectively, and suppose $H / N$ has $\mathbb{R}$-rank at least 2. Then $H / N$ and $H^{\prime} / N^{\prime}$ are locally isomorphic.

Proof. The orbit space of $\Gamma$ on $G / H$ is the same as that of $H$ on $G / \Gamma$ and, since $\Gamma$ is essentially free on $G / H, H$ will be essentially free on $G / \Gamma$. By the results of 
[6], we have an orbit equivalence $\theta: G / \Gamma \rightarrow G^{\prime} / \Gamma^{\prime}$ of the $H$ and $H^{\prime}$-actions on these spaces. Let $H_{0} \subset H, H_{0}^{\prime} \subset H^{\prime}$ be the connected components of the identity. Let $\alpha_{1}: G / \Gamma \times H \rightarrow H / H_{0}$ be the cocycle defined by restricting projection of $H$ onto $H / H_{0}$ and let $\alpha_{2}: G / \Gamma \times H \rightarrow H^{\prime} / H_{0}^{\prime}$ be the cocycle defined by

$$
\alpha_{2}(x, h)=p^{\prime} \beta(x, h),
$$

where $\beta: G / \Gamma \times H \rightarrow H^{\prime}$ is the cocycle defined by the orbit equivalence $\theta$ and $p^{\prime}: H^{\prime} \rightarrow H^{\prime} / H_{0}^{\prime}$ is projection. We thus have a cocycle $\left(\alpha_{1}, \alpha_{2}\right)$ taking values in $H / H_{0} \times H^{\prime} / H_{0}^{\prime}$ and we let $F \subset H / H_{0} \times H^{\prime} / H_{0}^{\prime}$ be the Mackey range of this cocycle. (Here, as usual, we abuse notation and identify a transitive Mackey range with a stabilizer of this transitive action.) Let $\lambda$ be a cocycle equivalent to $\left(\alpha_{1}, \alpha_{2}\right)$ taking values in $F$. Since $H_{0}, H_{0}^{\prime}$ are ergodic on $G / \Gamma, G^{\prime} / \Gamma^{\prime}$ respectively (by Moore's theorem [13]), $\alpha_{1}$ and $\alpha_{2}$ have Mackey range $H / H_{0}, H^{\prime} / H_{0}^{\prime}$ respectively, and so we have an $H$-map of extensions of $G / \Gamma, G / \Gamma \times{ }_{\lambda} F \rightarrow G / \Gamma \times H / H_{0}$. The $H$-space $G / \Gamma \times H / H_{0}$ clearly has the same orbit space as the $H_{0}$ action on $G / \Gamma$, and it is easy to see that the $H$-space $G / \Gamma \times_{\lambda} F$ has the same orbit space as the $H_{0}$-action on a finite ergodic extension $Y$ of $G / \Gamma$. On the other hand, via $\theta$, we can identify $\left(\alpha_{1}, \alpha_{2}\right), \lambda$ as cocycles on the $H^{\prime}$-space $G^{\prime} / \Gamma^{\prime}$ and $G / \Gamma \times{ }_{\lambda} F$ is thus easily seen to be orbit equivalent to an $H^{\prime}$-space which factors to $G^{\prime} / \Gamma^{\prime} \times H^{\prime} / H_{0}^{\prime}$.

In conclusion, we have orbit equivalent $H_{0}, H_{0}^{\prime}$-spaces which are essentially free and with finite invariant measure. Let $N_{0}$ be the maximal normal amenable subgroup of $H_{0}$ and suppose $L \subset H_{0}$ is a normal subgroup with $N_{0} \subset L$ and $N_{0} \neq L$. Then, by Moore's theorem [13], $L$ is ergodic on $G / \Gamma$ and it is easy to deduce from this that the space of ergodic components of $L$ on $Y$ is finite. But since $L \subset H_{0}$ is normal, $H_{0}$ acts on this finite set, and the $H_{0}$ action is transitive, since $H_{0}$ is ergodic on $Y$. From the connectedness of $H_{0}$, the set is a point so $L$ is ergodic on $Y$. Thus, the $H_{0}$ action satisfies the irreducibility condition in theorem 3.3, and an application of theorem 3.3 then completes the proof.

Remarks. (i) The proof and the results of [6] show that the theorem is true if we only assume that the $\Gamma$ action on $G / H$ and the $\Gamma^{\prime}$ action on $G^{\prime} / H^{\prime}$ are stably orbit equivalent in the sense of [6].

(ii) If $G$ and $H$ are algebraic, $H \supset Z(G)$ (the centre of $G$ ) and $H$ does not contain a normal non-central subgroup, then $\Gamma / \Gamma \cap Z(G)$ acts essentially freely on $G / H$. To see this, observe that for $\gamma \in \Gamma,\{x \in G / H \mid x \gamma=x\}$ is a closed variety, and hence if it has positive measure $\gamma$ fixes all of $G / H$ which implies $\gamma \in Z(G)$.

As an example of an application of theorem 4.1, we have the following.

THEOREM 4.2. (a) The actions of $\operatorname{SL}(n, \mathbb{Z})$ on the projective spaces $\mathbb{P}^{n-1}$ are mutually non-orbit equivalent as $n$ varies, $n \geq 2$.

(b) The actions of $\mathrm{SL}(n, \mathbb{Z})$ on $\mathbb{R}^{n}$ are mutually non-orbit equivalent as $n$ varies, $n \geq 2$.

(c) For a fixed $n \geq 4$, let $G_{n, k}$ be the Grassman variety of $k$-planes in $\mathbb{R}^{n}$. Then the actions of $\mathrm{SL}(n, \mathbb{Z})$ on $G_{n, k}$ are mutually non-orbit equivalent as $k$ varies, $1 \leq k \leq[n / 2]$. 
Proof. If we compare the actions of SL $(n, \mathbb{Z})$ on $\mathbb{P}^{n-1}$ and SL $(p, \mathbb{Z})$ on $\mathbb{P}^{p-1}$ for $n \neq p, n, p \geq 3$, then we can apply theorem 4.1 (and remark (ii) above), since the semi-simple part of the stabilizer of a point in $\mathbb{P}^{n-1}$ has $\mathbb{P}$-rank $n-2$. However, for $n=2$, the stabilizer is amenable and hence the $\operatorname{SL}(2, \mathbb{Z})$ action on $\mathbb{P}^{1}$ will also be amenable. (This follows from the fact that if $P$ is amenable, $G / P$ is an amenable $G$-space [20] and hence an amenable $\Gamma$-space [22].) Since the SL $(n, \mathbb{Z})$ action on $\mathbb{P}^{n-1}$ is not amenable for $n \geq 3$ [22, proposition 3.4], the proof of $(a)$ is complete. The proofs of $(b)$ and $(c)$ are similar.

We remark that theorem 4.2 remains true if we replace $\operatorname{SL}(n, \mathbb{Z})$ by any lattice in $\operatorname{SL}(n, \mathbb{R})$. Furthermore, the same argument yields similar results for actions on more general real and complex flag manifolds. We leave the details to the reader.

As in [24], theorems 3.1 and 3.3 can be extended to ergodic actions of lattices in connected groups. Thus, if $\Gamma_{i}$ are lattices in groups $H_{i}$ as in theorem 3.1 and the $\Gamma_{i}$ have free ergodic orbit equivalent actions with finite invariant measure, then the $H_{i} / N_{i}$ are isomorphic. The proof follows by applying theorem 3.1 to the actions of $H_{i}$ induced from $\Gamma_{i}$ [22]. For example, we have the following.

THEOREM 4.3. Let $\Gamma_{n}$ be the semi-direct product of $\mathrm{SL}(n, \mathbb{Z})$ with $\mathbb{Z}^{n}, n \geq 2$, where the former acts on the latter by matrix multiplication. Then for $i \neq j, \Gamma_{i}$ and $\Gamma_{i}$ do not have orbit equivalent finite measure preserving free ergodic actions.

In [25] it is shown that for $n \geq 3, \Gamma_{n}$ and $\operatorname{SL}(n, \mathbb{Z}) \times \mathbb{Z}^{n}$ do not have orbit equivalent finite measure preserving free weakly mixing actions.

\section{Concluding remarks}

(a) It would be of interest to extend theorem 3.4 to arbitrary (i.e. not product actions) finite measure preserving free ergodic actions of product groups, or perhaps semi-direct product groups. For example, if $H_{i}$ is a semi-direct product of $G_{i}$ and normal $A_{i}, i=1,2$, where $A_{i}$ is amenable and $G_{i}$ is a suitable semi-simple group, when can one deduce that orbit equivalence of the $H_{i}$ actions implies equivalence of associated $G_{i}$-actions (e.g. the actions of $G_{i}$ obtained by restricting the $H_{i}$ actions; actions of $G_{i}$ induced on the space of $A_{i}$-ergodic components, etc.))?

(b) One can also ask about the converse assertion in ( $a$ ). Namely if the $G_{i}$-actions are orbit equivalent (and hence under suitable hypotheses conjugate modulo an automorphism) are the $H_{i}$-actions orbit equivalent? When $G_{i}=\{e\}$ this, of course, follows from hyperfiniteness of amenable actions [16], [3] and Dye's theorem [4].

(c) Finally, one would like to know to what extent the hypotheses that the $\mathbb{R}$-rank be at least 2 are needed. A start in this direction appears in [26].

This reasearch was partially supported by NSF grant MCS79-05036, The Sloan Foundation, and the National Academy of Sciences, USA. The author is a Sloan Foundation Fellow.

\section{REFERENCES}

[1] A. Borel. Density properties for certain subgroups of semi-simple groups without compact factors. Annals of Math. 72 (1960), 179-188. 
[2] A. Borel \& J. Tits. Groupes reductifs. Publ. Math. I.H.E.S. 27 (1965), 55-150.

[3] A. Connes, J. Feldman \& B. Weiss. To appear.

[4] H. A. Dye. On groups of measure preserving transformations, I. Amer. J. Math. 81 (1959), 119-159.

[5] J. Feldman \& C. C. Moore. Ergodic equivalence relations, cohomology, and Von Neumann algebras, I. Trans. Amer. Math. Soc. 234 (1977), 289-324.

[6] J. Feldman, P. Hahn \& C. C. Moore. Orbit structure and countable sections for actions of continuous groups. Advances in Math. 28 (1978), 186-230.

[7] H. Furstenberg. Boundary theory and stochastic processes on homogeneous spaces. In Harmonic Analysis on Homogeneous Spaces, pp. 193-229. Symp. in Pure Math. Williamstown, Mass., 1972.

[8] G. W. Mackey. Point realizations of transformation groups. Ill. J. Math. 6 (1962), 327-335.

[9] G. W. Mackey. Ergodic theory and virtual groups. Math. Ann. 166 (1966), 187-207.

[10] G. A. Margulis. Non-uniform lattices in semisimple algebraic groups. In Lie Groups and Their Representations (ed. I. M. Gelfand), pp. 371-553. Wiley: New York, 1975.

[11] G. A. Margulis. Discrete groups of motions of manifolds of non-positive curvature. Amer. Math. Society Translations 109 (1977), 33-45.

[12] G. A. Margulis. Arithmeticity of irreducible lattices in semisimple groups of rank greater than 1. Appendix to Russian translation of M. Ragunathan. Discrete Subgroups of Lie Groups. Mir: Moscow, 1977. (In Russian.)

[13] C. C. Moore. Ergodicity of flows on homogeneous spaces. Amer. J. Math. 88 (1966), 154-178.

[14] G. D. Mostow. Quasi-conformal mappings in $n$-space and the rigidity of hyperbolic space forms. Publ. Math. I.H.E.S. 34 (1967), 53-104.

[15] G. D. Mostow. Strong Rigidity of Locally Symmetric Spaces. Annals of Math. Studies, no. 78. Princeton Univ. Press: Princeton, N.J., 1973.

[16] D. Ornstein \& B. Weiss. To appear.

[17] A. Ramsay. Virtual groups and group actions. Advances in Math. 6 (1971), 253-322.

[18] C. Series. The Rohlin tower theorem and hyperfiniteness for actions of continuous groups. Israel J. Math. 30 (1978), 99-112.

[19] C. Series. Foliations of polynomial growth are hyperfinite. Israel J. Math. 34 (1979), 245-258.

[20] R. J. Zimmer. Amenable ergodic group actions and an application to Poisson boundaries of random walks. J. Funct. Anal. 27 (1978), 350-372.

[21] R. J. Zimmer. Orbit spaces of unitary representations, ergodic theory, and simple Lie groups. Annals of Math. 106 (1977), 573-588.

[22] R. J. Zimmer. Induced and amenable ergodic actions of Lie groups. Ann. Sci. Ec. Norm. Sup. 11 (1978), 407-428.

[23] R. J. Zimmer. An algebraic group associated to an ergodic diffeomorphism. Comp. Math. 43 (1981), 59-69.

[24] R. J. Zimmer. Strong rigidity for ergodic actions of semisimple Lie groups. Annals of Math. 112 (1980), 511-529.

[25] R. J. Zimmer. On the cohomology of ergodic actions of semisimple Lie groups and discrete subgroups. Amer. J. Math. (in the press).

[26] R. J. Zimmer. On the Mostow rigidity theorem and measurable foliations by hyperbolic space. Preprint. 